\title{
Temporospatial dissociation of Pe subcomponents for perceived and unperceived errors
}

\author{
Tanja Endrass*, Julia Klawohn, Julia Preuss and Norbert Kathmann
}

Institute for Psychology, Humboldt University Berlin, Berlin, Germany

\section{Edited by:}

Claudia Danielmeier, Radboud

University Nijmegen, Netherlands

Reviewed by:

K. Richard Ridderinkhof, University

of Amsterdam, Netherlands

Wim Notebaert, Ghent University,

Belgium

${ }^{*}$ Correspondence:

Tanja Endrass, Institute for

Psychology, Humboldt University

Berlin, Rudower Chaussee 18,

12489 Berlin, Germany.

e-mail: endrass@gmail.com
Previous research on performance monitoring revealed that errors are followed by an initial fronto-central negative deflection (error-related negativity, ERN or $\mathrm{Ne}$ ) and a subsequent centro-parietal positivity (error positivity, Pe). It has been shown that error awareness mainly influences the $\mathrm{Pe}$, whereas the ERN seems unaffected by conscious awareness of an error. The aim of the present study was to investigate the relation of ERN and Pe to error awareness in a visual size discrimination task in which errors are not elicited by impulsive responding but by perceptual difficulty. Further, we applied a temporospatial principal component analysis (PCA) to examine whether the temporospatial subcomponents of the Pe would differentially relate to error awareness. Event-related potential (ERP) results were in accordance with earlier studies: a significant error awareness effect was found for the $\mathrm{Pe}$, but not for the ERN. Interestingly, a modulation with error perception on correct trials was found: correct responses considered as incorrect had larger correct-related negativity (CRN) and lager Pe amplitudes than correct responses considered as correct. The PCA yielded two relevant spatial factors accounting for the Pe (latency $300 \mathrm{~ms}$ ). A temporospatial factor characterized by a centro-parietal positivity varied significantly with error awareness. Of the two temporospatial factors corresponding to ERN and CRN, one factor with central topography varied with response correctness and subjective error perception on correct responses. The PCA results indicate that the error awareness effect is specifically related to the centro-parietal subcomponent of the Pe. Since this component has also been shown to be related to the importance of an error, the present variation with error awareness indicates that this component is sensitive to the salience of an error and that salience secondarily may trigger error awareness.

Keywords: error awareness, error-related negativity, error positivity, principal component analysis, PCA

\section{INTRODUCTION}

Performance monitoring is an essential prerequisite for adaptive behavior and implements adjustment processes, such as error detection and subsequent post-error slowing. Over the past years numerous psychophysiological and neuroimaging studies investigated the neural basis of performance monitoring and error processing. Event-related potential (ERP) studies identified a fronto-central negativity that emerges shortly after the execution of incorrect responses, the error-related negativity (ERN, Gehring et al., 1993) or error negativity (Ne, Falkenstein et al., 1991). Sometimes a smaller negative deflection is also observed for correct responses, the correct-related negativity (CRN, Ford, 1999; Vidal et al., 2000). The ERN is followed by the error positivity (Pe), a centro-parietal positive deflection that peaks between 200 and $400 \mathrm{~ms}$ after response onset (Falkenstein et al., 1991, 2000; Overbeek et al., 2005). These components are considered to indicate error-related brain activity but it is not fully clear whether they reflect functionally dissociate aspects of error processing. While source localization studies suggest that the ERN is generated in the anterior cingulate cortex (ACC), or more precisely, Hyphenation: gene-rated in the posterior medial frontal cortex (pMFC, Dehaene et al., 1994; Van Veen and Carter, 2002; Debener et al., 2005), the source of the Pe is more difficult to determine, and heterogeneous results were obtained (Herrmann et al., 2004; O'Connell et al., 2007; Vocat et al., 2008).

Although it is broadly agreed that the ERN and Pe are linked to error commission, their functional significance still remains to be clarified. Currently, the ERN is regarded as a negative reinforcement learning signal (Holroyd and Coles, 2002), or as an index for response conflict or error likelihood (Botvinick et al., 2001; Yeung et al., 2004; Brown and Braver, 2007, 2008). These models assume that the ERN or its underlying ACC activity plays a key role in the recruitment of cognitive control in response to erroneous actions in order to prevent future errors (Ridderinkhof et al., 2004; Ullsperger et al., 2004). Since the Pe most consistently varies with motivational salience and subjective error perception, it is considered to reflect evaluative aspects of error processing (Falkenstein et al., 2000; Overbeek et al., 2005).

The sensitivity to error awareness is perhaps the most important distinction between both error-related ERP components. Results were rather consistent in that reduced Pe components were reported for unaware/unperceived compared to aware/perceived errors (Nieuwenhuis et al., 2001; Endrass et al., 2005, 2007; O'Connell et al., 2007; Shalgi et al., 2009; Dhar et al., 2011; Wessel et al., 2011). Whereas the Pe seems clearly sensitive to error awareness, the majority of studies found no effect 
on the ERN (Nieuwenhuis et al., 2001; Endrass et al., 2005, 2007; O'Connell et al., 2007; Shalgi et al., 2009; Dhar et al., 2011). However, there is some evidence that an error awareness effect may also be present for the ERN (Maier et al., 2008; Hewig et al., 2011; Wessel et al., 2011). Interestingly, a recent study provided evidence that ERN and Pe reflect dissociate aspects of error processing in a choice selection task. In that task errors were compared between a masking and a conflict condition. While the ERN was substantially reduced in the masking condition, the Pe varied with error awareness (Hughes and Yeung, 2011). Neuroimaging studies examining error awareness reported that the activity of the anterior insula was significantly modulated by conscious error perception and suggested this region to indirectly contribute to the emergence of a Pe (Hester et al., 2005; Klein et al., 2007). The same region was shown to be sensitive to interoceptive awareness (Critchley et al., 2004, 2005), and is reliably associated with autonomous activation (Ullsperger et al., 2010). Therefore, it was suggested that error awareness emerges when information for error commission aggregates within the salience network, and its presence is to some extent indicated by the Pe (Ullsperger et al., 2010).

Additional evidence that the Pe may be related to saliency of incorrect response can be derived from the fact that topography and time course of the Pe displays similarities to the P300 or P3b (Falkenstein et al., 1999; Leuthold and Sommer, 1999; Hajcak et al., 2003; Overbeek et al., 2005; Ridderinkhof et al., 2009). The P3b is a stimulus-locked positive brain potential that is elicited in response to rare and motivationally significant events (Picton, 1992; Nieuwenhuis et al., 2005). This component has been considered to reflect the response of the locus coeruleus-norepinephrine system to the outcome of internal decision-making processes (Nieuwenhuis et al., 2005). Errors are rare and salient events that trigger a cascade of central nervous and autonomous changes and are considered as events that are motivationally significant and thus elicit a P3b (e.g., Hajcak et al., 2003; O'Connell et al., 2007; Wessel et al., 2011). Hence, the Pe might be understood as a P3b to error commission. Using principal components analysis (PCA) it has been shown that the Pe is composed of two different subcomponents: a fronto-central component that shares spatial distribution with the ERN and a centro-parietal component that is similar to the P300 (Arbel and Donchin, 2009). While emphasis on accuracy did not cause significant variation of the fronto-central component, the centroparietal component was enlarged for this condition. These results highlight the association between the centro-parietal component of the Pe and error salience.

The aim of the current study is to investigate error awareness effects on ERN and Pe in a choice selection task. In this task errors are elicited due to perceptual difficulty instead of failures to withhold a response. Specifically, we were interested in the structure of the underlying processes of the Pe and conducted a temporospatial PCA (Dien, 2010a; Endrass et al., 2012). The aim was to disentangle overlapping electrophysiological activity captured in the Pe, and to examine the sensitivity of the subcomponents of ERN and Pe to error awareness. In the ERP analysis we expected to replicate earlier findings showing that error awareness selectively affects the Pe, but not the ERN. In addition, we examined more closely the effect of subjective error perception in correct responses. With the PCA analysis we intended to replicate the previously identified subcomponents of the Pe (Arbel and Donchin, 2009) and to examine whether these subcomponents would be differentially sensitive to error awareness. Since the centro-parietal subcomponent of the Pe was associated with error salience, we expected only this component to be sensitive to error awareness.

\section{MATERIALS AND METHODS PARTICIPANTS}

Seventeen healthy undergraduate students (nine female, 20-30 years, mean \pm SD, 23.4 years \pm 3.1 ) of the Humboldt-University Berlin voluntarily took part in the present experiment ${ }^{1}$. The participants either received monetary remuneration or class credit points for their participation. All of them were in good health, with no history of psychiatric or neurological disease, and had normal or corrected to normal vision. In accordance to the ethical guidelines of the Declaration of Helsinki, written informed consent was enquired before the experimental procedures started.

\section{TASK AND EXPERIMENTAL PROCEDURE}

A visual size discrimination task was employed with a display showing two dots to compare. Participants were seated at a distance of $70 \mathrm{~cm}$ in front of a 19-in computer screen. Each stimulus comprised a standard (visual angle of $2.4^{\circ}$ ) and a comparison dot that varied in size with task difficulty $\left(2.5,2.6\right.$, and $2.7^{\circ}$ in the difficult, intermediate and easy condition, respectively). Both dots were presented in white color against dark background left and right to a fixation cross (distance $1.4^{\circ}$ ).

Each trial started with a fixation cross displayed for 600-1000 ms, followed by the stimulus array presented for $500 \mathrm{~ms}$. After that, the screen turned blank for $1000 \mathrm{~ms}$. Participants were asked to select the larger dot by a left or right response button press. Instruction equally emphasized the importance of speed and accuracy. Subsequently, a display requesting for accuracy ratings was shown for $1000 \mathrm{~ms}$. Participants were instructed to evaluate whether the previous response was correct, incorrect, or they were unsure. The experiment comprised a total number of 832 trials administered in four blocks. The target stimuli were presented equally frequent at both sides of the screen and subjects were informed that always one of the dots was larger than the other. The hard and intermediate conditions encompassed 208 trials each and 416 trials were presented for the easy condition, all distributed evenly over the four blocks, and displayed in pseudo-randomized order. A larger number of easy trials were presented to obtain a reasonable number of error trials in that condition since the goal was to compare errors between difficulty levels. The completion of the whole experimental task

\footnotetext{
${ }^{1}$ The data presented here were also included in a previous study from our group that focused on differences in post-response potentials following errors and correct responses and addressed the question whether ERN and CRN reflect different aspects of performance monitoring (Endrass et al., 2012). Specifically, the previous analysis compared correct and incorrect responses in easy, intermediate, and difficult condition. The results presented here were not reported in the previous report and data analysis followed a different rational.
} 
lasted about 60 min including a practice block and short breaks between blocks.

\section{ELECTROENCEPHALOGRAPHIC RECORDING AND ANALYSIS}

The electroencephalogram (EEG) was recorded from 63 electrodes, with 61 equidistant electrode positions including $\mathrm{Cz}$ as recording reference placed in an electrode cap (EasyCap, Herrsching, Germany) and two external electrodes located below the left and right eye. The ground electrode was placed below T1. It was ensured that electrode impedances were below $5 \mathrm{k} \Omega$. EEG was continuously recorded at a sampling rate of $500 \mathrm{~Hz}$. Amplifiers (BrainAmp BrainProducts, Gilching Germany) used a high-pass filter with a time constant of $10 \mathrm{~s}(0.0159 \mathrm{~Hz})$ and a low-pass filter set at $250 \mathrm{~Hz}$.

Responses were obtained from two force-sensitive response devices. Force was continuously recorded in two separate recording channels together with the EEG signals. Devices were calibrated to $200 \mu \mathrm{V}$ equaling $1 \mathrm{~N}$. Participants were instructed to rest their index fingers on the devices, and to press them in order to respond. For data analysis, first, the mean activity of a $200 \mathrm{~ms}$ pre-stimulus baseline interval was subtracted. Then, an algorithm searched for amplitude maxima exceeding predefined thresholds for partial responses (minimum of $0.25 \mathrm{~N}$ equaling $50 \mu \mathrm{V}$ ) and full responses (minimum of $0.5 \mathrm{~N}$ ). Given an above-threshold activity, response onsets were marked at the initial force onset as indicated by an amplitude change of $20 \mu \mathrm{V}$ within $20 \mathrm{~ms}$. This method allowed to detect both partial responses as well as full responses and to distinguish them for further analyses. In the present study, only purely correct responses which were not preceded by any partial incorrect responses within the same trial were included into analyses. Accordingly, error trials were only taken into account if they were not preceded by partial correct reactions within the trial.

For ERP analysis, EEG data were filtered off-line with a lowpass filter set at $40 \mathrm{~Hz}(12 \mathrm{db} / \mathrm{Oct})$ as well as with a $50 \mathrm{~Hz}$ notch filter, and re-referenced to average reference. Correction for eye-movements and blink artifacts was applied using the multiple source eye correction method (Berg and Scherg, 1994) implemented in BESA5 (Brain Electrical Source Analysis, MEGIS Software GmbH, Gräfelfing, Germany). Epochs of 1200 ms starting $200 \mathrm{~ms}$ before the first response in each trial were obtained from continuous EEG data. Epochs were baseline corrected using the $200 \mathrm{~ms}$ pre-response window. Segments containing amplitude changes exceeding $200 \mu \mathrm{V}$, or voltage steps of more than $100 \mu \mathrm{V}$ between consecutive data points were rejected from further analysis. For each participant, four averages were computed: correct responses rated as correct (perceived correct) or incorrect (unperceived correct) as well as erroneous responses rated as correct (unperceived errors) or incorrect (perceived errors). Although a behavioral pilot study (10 participants) indicated that it would be possible to obtain a sufficient number of perceived and unperceived errors in all three difficulty levels, only very few participants $(N=8)$ had a minimum number of six errors in each condition. Therefore, the factor task difficulty could not be taken into account for this analysis. For visual presentation, grand averages were filtered with a $15 \mathrm{~Hz}$ low-pass filter.
For ERP analysis, ERN and CRN amplitudes were quantified as mean amplitudes between 60 and $140 \mathrm{~ms}$ post-response at frontocentral electrode sites $(\mathrm{Fz}, \mathrm{FCz}$, and $\mathrm{Cz})$. The Pe was measured as mean amplitudes at the electrodes $\mathrm{Cz}, \mathrm{CPz}$, and $\mathrm{Pz}$ between 300 and $500 \mathrm{~ms}$ after response onset. ERP amplitudes were statistically analyzed with repeated measurement ANOVAs with the factors Response Type (correct vs. incorrect), Response Rating (response perceived as correct vs. incorrect) and Electrode Site. Greenhouse-Geisser correction was applied when appropriate. All statistical analyses for the present study were conducted with IBM SPSS Statistics (Version 19.0, Chicago).

A covariance-based two-step temporospatial PCA was computed on individual response-locked ERP averages using the ERP PCA Toolkit 2.06 (Dien, 2010a,b). In accordance with Dien et al., 2005, a covariance matrix and Kaiser normalisation was applied. The temporospatial PCA extracts linear combinations of data that distinguish patterns of electrocortical activity across all time points and recording sites (see also Dien and Frishkoff, 2005). The temporospatial sequence of analyses was chosen since this was found to be most effective in simulation studies (Dien, 2010b). First, the temporal PCA was computed using the individual averages of each participant over all 63 electrodes, for correct and incorrect responses in the two response rating conditions (perceived as correct vs. incorrect). Each dataset consisted of 600 time points (-200 to $1000 \mathrm{~ms})$. A scree plot was used to limit extracted factors in number, resulting in the promax rotation that yielded 19 temporal factors. Then, in order to analyze their spatial distribution, separate spatial PCA (infomax rotation) was applied to each temporal factor. In total, the temporospatial PCA yielded 76 factor combinations (four spatial factors extracted for each of the 19 temporal factors). Only those temporospatial factors that uniquely accounted for more than $1 \%$ of the total variance in the data were included in further analyses (Kayser and Tenke, 2005; Foti et al., 2009, 2011). Note that the amount of explained variance by one factor is related to the total variance in the data, i.e., all time points and all electrodes. Factor scores of these factors were plotted as "virtual ERPs" and averaged for both response types and rating conditions. The temporal factors corresponding to ERN/CRN and Pe, as our ERP components of interest, were selected by temporal characteristics of the PCA waveforms (Dien et al., 2005, 2010; Foti et al., 2011). The resulting factor scores were submitted to statistical analysis using repeated measurement ANOVA with the factors Response Type and Response Rating.

Error and correct awareness were determined as the percentages of errors and correct responses that were adequately perceived as incorrect or correct, respectively. Behavioral data were analyzed by repeated-measurement ANOVAs. Awareness was analyzed with the factors Response Type (error vs. correct) and Difficulty (easy, intermediate vs. difficult). Analysis of reaction time data involved the factors Response Type and Response Rating. Post-error adjustment effects were analyzed in terms of subsequent reaction time (post-error slowing) and response correctness. For post-error slowing, correct reaction times following perceived and unperceived correct and incorrect responses were analyzed by an ANOVA with the factors Preceding Response Type and Preceding Response Rating of the preceding response. To examine whether there was a relative 
increase of response correctness after erroneous responses (posterror correctness), the percentage of correct responses following perceived and unperceived errors and correct responses was assessed.

\section{RESULTS}

\section{BEHAVIORAL DATA}

Main behavioral results are presented in Table 1. On average, participants committed $16.1 \%( \pm S D 5.2)$ errors and $80.2 \%$ $( \pm \mathrm{SD} 6.6)$ of all responses were correct. The percentage of correct perceptions, i.e., the amount of correctly classified correct and incorrect responses, varied with Response Type, $\left[F_{(1,16)}=\right.$ 142.59, $\left.p<0.001, \eta^{2}=0.90\right]$, and Difficulty, $\left[F_{(2,32)}=17.27\right.$, $\left.p<0.001, \varepsilon=0.88, \eta^{2}=0.52\right]$. The amount of correct perceptions was significantly higher for correct than incorrect responses (mean \pm SD, $93.2 \% \pm 3.3$ vs. $51.5 \% \pm 13.6$ ) and for easy and intermediate compared to difficult trials (mean $\pm \mathrm{SD}, 73.6 \%$ $\pm 8.7,76.7 \% \pm 8.2$, vs. $66.8 \% \pm 6.6), p<0.008$. Further, these effects resulted in a significant interaction of both factors, $\left[F_{(2,32)}=3.83, p=0.044, \varepsilon=0.79, \eta^{2}=0.19\right]$. While relative error perception was lower for easy and difficult compared to intermediate trials (mean $\pm \mathrm{SD}, 50.9 \% \pm 17.7,44.5 \% \pm 14.0$, vs. $58.9 \% \pm 15.3$, easy, difficult and intermediate condition, respectively), $p=0.025$ and $p=0.007$, easy and difficult conditions did not differ, $p=0.335$. For correct responses, more correct perceptions were found for easy and intermediate compared to difficult trials, $p<0.001$ and $p=0.002$ (mean $\pm \mathrm{SD}, 96.3 \% \pm$ $2.1,94.5 \% \pm 4.1$, vs. $89.0 \% \pm 6.2$, easy, intermediate, and difficult condition, respectively) ${ }^{2}$.

The analysis of reaction time data revealed a main effect of Response Type, $\left[F_{(1,16)}=6.40, p=0.022, \eta^{2}=0.29\right]$, that was qualified by an interaction of Response Type and Response Rating, $\left[F_{(1,16)}=12.29, p=0.003, \eta^{2}=0.43\right]$. Overall, error reaction times were faster than correct reaction times. Mean reaction times were faster for perceived errors compared to unperceived errors, $p=0.047$ (mean $\pm \mathrm{SD}, 336 \mathrm{~ms} \pm 50,351 \mathrm{~ms} \pm 60$ ), and for perceived correct compared to unperceived correct responses, $p=0.001$ (mean $\pm \mathrm{SD}, 346 \mathrm{~ms} \pm 42,364 \mathrm{~ms} \pm 49$ ).

Concerning post-error slowing, reaction times of correct responses following perceived and unperceived errors as well as those following perceived and unperceived correct responses were analyzed (mean values in Table 1). Only the main effect of Preceding Response Rating was significant, $\left[F_{(1,16)}=17.06\right.$, $\left.p=0.001, \eta^{2}=0.52\right]$, but neither the main effect of Preceding Response Type nor the interaction were significant, $F<1$. Posthoc comparisons indicate that reaction times were slower in trials following reactions that were subjectively perceived as incorrect

\footnotetext{
${ }^{2}$ Since error awareness varied between difficulty conditions and a larger number of trials were presented in the easy condition, the absolute numbers of easy, intermediate, and difficult trials were compared between perceived and unperceived errors. An interaction between Difficulty and Response Rating was found at trend level, $\left[F_{(2,16)}=3.55, p=0.058, \eta^{2}=0.18\right]$. While the number of perceived and unperceived errors did not differ in the easy (31.0 vs. 26.3 ) and difficult condition (24.9 vs. 29.5$)$, significantly more perceived than unperceived error occurred in the intermediate condition (19.6 vs. 13.5), $p=0.025$. Hence, the number of errors did not differ between easy and difficult condition for both rating types.
}

Table 1 | Behavioral results and amplitudes of response-locked negativities (ERN/Ne and CRN) at electrode FCz in the three difficulty conditions (means and standard deviations).

\begin{tabular}{|c|c|c|c|c|c|c|}
\hline & \multicolumn{6}{|c|}{ Task difficulty } \\
\hline & \multicolumn{2}{|c|}{ Easy } & \multicolumn{2}{|c|}{ Intermediate } & \multicolumn{2}{|c|}{ Difficult } \\
\hline & $M$ & SD & $M$ & SD & $M$ & SD \\
\hline Error rates (\%) & 14.9 & 6.1 & 18.1 & 7.2 & 31.3 & 5.9 \\
\hline Error RT (ms) & 309 & 67 & 321 & 60 & 341 & 60 \\
\hline Correct RT (ms) & 338 & 46 & 340 & 47 & 345 & 50 \\
\hline Error awareness (\%) & 47.3 & 19.1 & 49.6 & 16.3 & 37.6 & 15.4 \\
\hline Correct awareness (\%) & 94.3 & 3.5 & 91.8 & 6.2 & 81.9 & 10.1 \\
\hline Error correction (\%) & 32.8 & 17.2 & 29.9 & 16.6 & 16.6 & 11.1 \\
\hline ERN/Ne amplitude $(\mu \mathrm{V})$ & -4.11 & 2.81 & -4.22 & 2.60 & -3.21 & 1.91 \\
\hline CRN amplitude $(\mu \mathrm{V})$ & -1.91 & 2.24 & -2.17 & 2.17 & -2.22 & 1.89 \\
\hline
\end{tabular}

Note: $R T$, reaction time.

(mean $\pm \mathrm{SD}, 358 \pm 40$ ) compared to trials following reactions perceived as correct (mean $\pm \mathrm{SD}, 346 \pm 40$ ), irrespective of the actual correctness of the preceding response.

In contrast, the analysis of post-error correctness only revealed a significant main effect of Preceding Response Type, $\left[F_{(1,16)}=\right.$ 4.81, $p=0.043, \eta^{2}=0.23$ ]. Overall, accuracy was higher following incorrect responses (mean $\pm \mathrm{SD}, 82.7 \% \pm 7.3$ ) than following correct responses (mean $\pm \mathrm{SD}, 79.3 \% \pm 7.0$ ). The main effect of Preceding Response Rating and the interaction were not significant, $F<2.4$.

\section{ERP RESULTS}

ERPs and topographies for both response types (correct and incorrect responses) and rating conditions (perceived as correct and incorrect) are displayed in Figure 1. ERN and CRN are apparent at fronto-central electrode sites peaking about $100 \mathrm{~ms}$ following response onset. Pe is evident at central electrodes in the time range between 200 and $600 \mathrm{~ms}$ after response onset.

Statistical analysis of ERN/Ne and CRN amplitudes yielded significant main effects of Electrode, $\left[F_{(2,32)}=19.43, p<0.001\right.$, $\left.\varepsilon=0.59, \eta^{2}=0.55\right]$, and Response Type, $\left[F_{(1,16)}=11.92, p=\right.$ $\left.0.003, \eta^{2}=0.43\right]$. These main effects were further specified by a significant interaction of Electrode $\times$ Response Type, $\left[F_{(2,32)}=\right.$ 9.80, $\left.p=0.002, \varepsilon=0.73, \eta^{2}=0.38\right]$. While no main effect of Response Rating or interaction of Response Rating and Response Type was found, $F<2.14, p>0.163$, the three-way interaction reached statistical trend level, $\left[F_{(2,32)}=2.80, p=0.105\right.$, $\left.\varepsilon=0.60, \eta^{2}=0.149\right]$. Follow-up comparisons of the Electrode $\times$ Response Type interaction revealed significantly larger ERN than CRN amplitudes at FCz and $\mathrm{Cz}$, but not at $\mathrm{Fz}, p=0.004, p=$ 0.001 , and $p=0.149$, respectively. CRN amplitudes were largest at Fz compared to $\mathrm{FCz}$ and $\mathrm{Cz}, p=0.041$ and $p<0.001$, respectively. ERN amplitudes were larger at $\mathrm{FCz}$ compared to $\mathrm{Fz}$ and $\mathrm{Cz}, p<0.001$, but did significantly differ only between $\mathrm{FCz}$ and Cz. Post-hoc analysis of the three-way interaction revealed that perceived and unperceived errors did not differ at any electrode site, $p>0.48$. In contrast, unperceived correct responses elicited larger CRN amplitudes than perceived correct responses at FCz, $p=0.056$. 


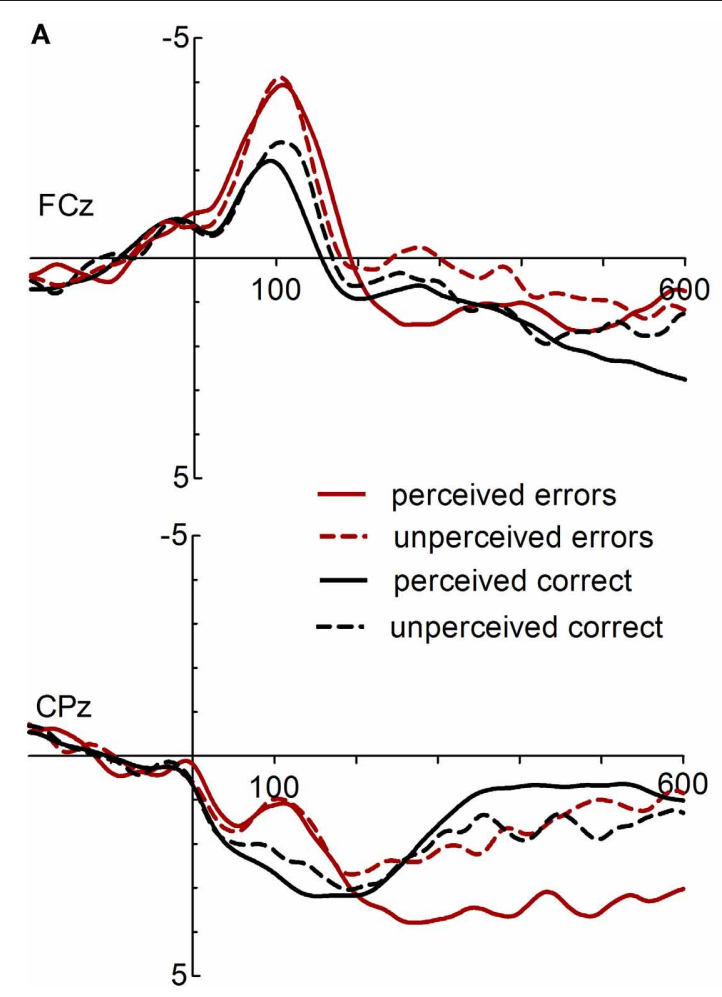

B

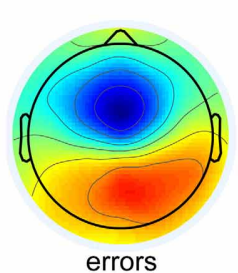

C
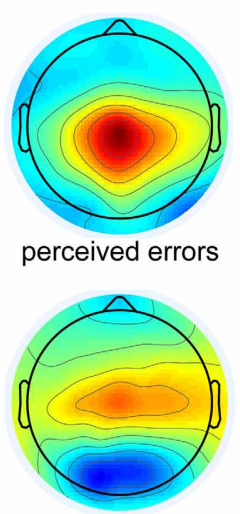

perceived correct

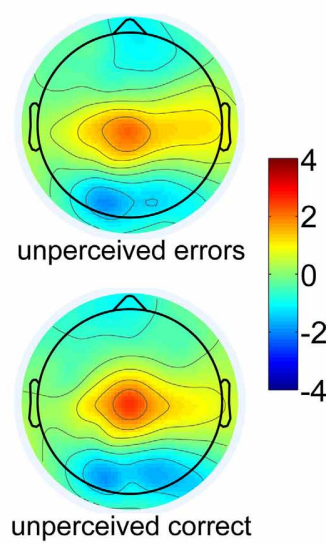

FIGURE 1 | (A) Response-locked ERPs following correct and incorrect response at electrodes FCz and CPz (left). (B) Topographies of the response-related negativities (ERN and CRN, $100 \mathrm{~ms}$ following response onset). (C) Topographies of the post-response positivity (Pe, $300 \mathrm{~ms}$ ).

Supplementary paired $t$-tests were used to examine the Response Rating effect for ERN and CRN amplitudes at several fronto-central and midline electrode sites (FCz, FC1, FC2, Fz, F1, $\mathrm{F} 2, \mathrm{Cz}, \mathrm{CPz}$, and $\mathrm{Pz}$ ). The comparison of perceived and unperceived errors yielded no significant difference at any electrode location, all comparisons $t<1, p>0.50$. Yet, larger CRN amplitudes were found for unperceived correct responses compared to perceived correct responses at $\mathrm{FCz}, t(16)=2.06, p=0.056$, FC1, $t(16)=4.71, p<0.001, \mathrm{~F} 1, t(16)=1.91, p=0.075, \mathrm{CPz}$, $t(16)=2.50, p=0.024$, and Pz, $t(16)=2.88, p=0.011$.

The analysis of $\mathrm{Pe}$ amplitudes revealed significant main effects of the factors Electrode, $\left[F_{(2,32)}=14.83, p=0.001, \varepsilon=\right.$ $\left.0.56, \eta^{2}=0.48\right]$, Response Type, $\left[F_{(1,16)}=22.47, p<0.001\right.$, $\left.\eta^{2}=0.58\right]$, and Response Rating, $\left[F_{(1,16)}=30.32, p<0.001\right.$, $\left.\eta^{2}=0.66\right]$. Further, the following interactions were significant: Response Type $\times$ Response Rating, $\left[F_{(1,16)}=11.08, p=0.004\right.$, $\left.\eta^{2}=0.41\right]$, and Electrode $\times$ Response Type, $\left[F_{(2,32)}=10.20\right.$, $\left.p=0.004, \varepsilon=0.56, \eta^{2}=0.40\right]$. Overall, Pe amplitudes were more positive for incorrect compared to correct responses. Thus, the effect of Response Rating is more pronounced for errors than for correct responses. Larger Pe amplitudes were found for perceived compared to unperceived errors (mean difference \pm SD, errors: $1.82 \mu \mathrm{V} \pm 1.26$ ), $p<0.001$, and for unperceived compared to perceived correct responses (mean difference \pm SD: $0.75 \mu \mathrm{V} \pm 1.07), p=0.011$. In fact, amplitudes did not differ between unperceived errors and unperceived correct responses. While the $\mathrm{Pe}$ is significantly more positive at $\mathrm{Cz}$ and $\mathrm{CPz}$ compared to $\mathrm{Pz}, p=0.004$ and $p<0.001$, the Pe is larger for incorrect than correct responses at $\mathrm{CPz}$ and $\mathrm{Pz}$, but not at $\mathrm{Cz}$, $p<0.001, p<0.001$, and $p=0.124$, respectively ${ }^{3}$.

\section{PCA RESULTS}

The application of the temporospatial PCA revealed 19 temporal factors and four spatial factors for each temporal factor,

\footnotetext{
${ }^{3}$ In addition, ERN and Pe amplitudes were analyzed with the factors Electrode, Response Rating and Difficulty (easy vs. difficult) in a subsample of 12 participants who committed at least six errors in each condition. For ERN amplitudes significant main effects of Electrode, $\left[F_{(2,22)}=5.12, p=0.015\right.$, $\left.\varepsilon=0.58, \eta^{2}=0.32\right]$, and Difficulty, $\left[F_{(1,11)}=24.40, p<0.001, \eta^{2}=0.69\right]$, were found. ERN amplitudes were smaller in the difficult compared to the easy condition. A statistical trend was found for the interaction of Electrode and Difficulty, $\left[F_{(2,22)}=3.68, p=0.077, \varepsilon=0.52, \eta^{2}=0.25\right]$. While the difficulty effect was significant at $\mathrm{FCz}$ and $\mathrm{Cz}(p<0.001)$, it only reached trend level at $\mathrm{Fz}(p=0.09)$. Importantly, a main effect of Response Rating or an interaction of Response Rating and Difficulty were not found, $\left[F_{(1,11)}<1\right]$. In addition, separate comparisons between perceived and unperceived errors in the easy and difficult condition did not reach significance $(p>0.3)$. The analysis of the Pe showed significant main effects of Electrode, $\left[F_{(2,22)}=13.33, p=0.001, \varepsilon=0.59, \eta^{2}=0.40\right]$, and Response Rating, $\left[F_{(1,11)}=25.87, p<0.001, \eta^{2}=0.56\right]$. Enhanced Pe amplitudes were elicited by perceived compared to unperceived errors. The main effect of Difficulty, $\left[F_{(1,11)}=1.41, p=0.25\right]$, or the interaction with Response Rating, $\left[F_{(1,11)}=0.09, p=0.76\right]$, were not significant. Separate comparisons indicate that the Pe enhancement for perceived errors was present in the easy $(p=0.028)$ and the difficult condition $(p=0.001)$.
} 
resulting in 76 factor combinations. The factors corresponding to the ERP components of interest were identified by their temporal characteristics, one peaking at $300 \mathrm{~ms}$ post-response and presumably reflecting the error positivity (TF2) and the other peaking at $104 \mathrm{~ms}$ corresponding to response-related negativities (TF3). Of the eight resulting temporospatial factors, two corresponding to the Pe (TF2SF1, TF2SF2) and two corresponding to the ERN/CRN (TF3SF1, TF3SF2) accounted for at least 1\% of the variance and were analyzed further.

In Figure 2, time course and topographies of the two spatiotemporal factors corresponding to the ERN and CRN are displayed. The first factor (TF3SF1, Figure 2A) had a central topography and uniquely accounted for $2.28 \%$ of variance in the solution whereas the second factor (TF3SF2, Figure 2B) displayed a frontal negativity and a parietal positivity and accounted for $1.82 \%$ of variance. The central factor (TF3SF1) was more negative for errors than correct responses as revealed by a significant main effect of Response Type, $\left[F_{(1,16)}=25.62, p<0.001\right.$, $\left.\eta^{2}=0.62\right]$. Although the main effect of Response Rating and the interaction with Response Type only reached a statistical

A TF3SF1: central factor

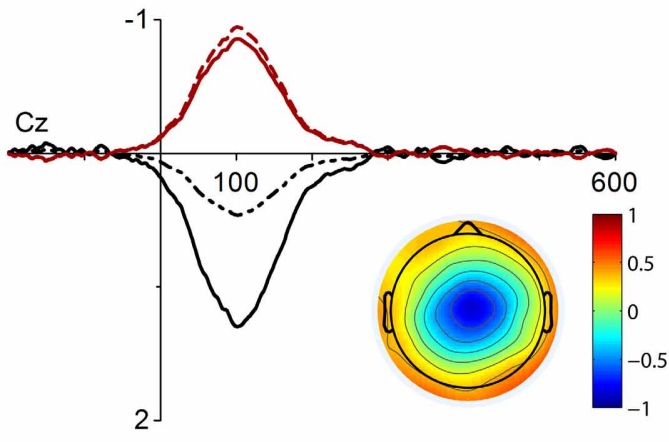

B TF3SF2: frontal factor

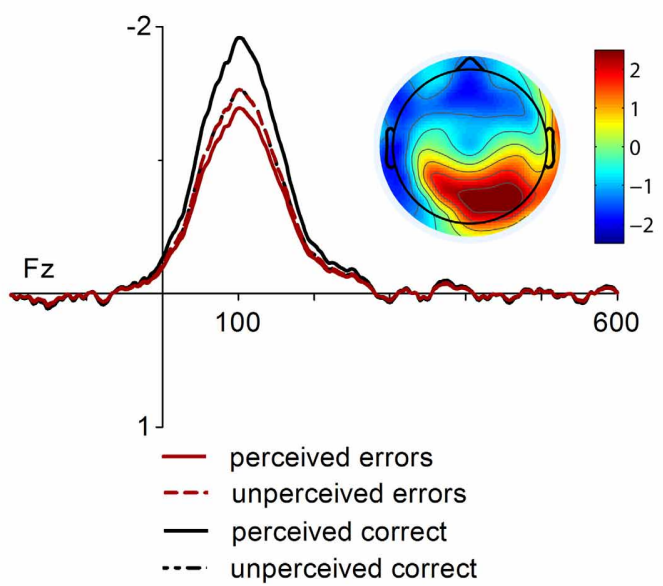

FIGURE 2 | "Virtual ERPs" and topographic maps (at $100 \mathrm{~ms}$ ) of the spatial factors contributing to ERN and CRN. The waveforms are presented at electrodes $\mathrm{Cz}$ and $\mathrm{Fz}$ where factor scores were at maximum. Waveforms and topographies of the central factor (A. TF3SF1) and the frontal factor (B. TF3SF2) are depicted.

trend, $\left[F_{(1,16)}=3.46, p=0.081, \eta^{2}=0.18\right]$ and $\left[F_{(1,16)}=3.97\right.$, $\left.p=0.064, \eta^{2}=0.20\right]$, follow-up comparisons for the interaction were conducted. While factor scores for perceived and unperceived errors did not differ, $p=0.75$, a significant awareness effect was found for correct responses, $p=0.003$. Factor scores of the frontal factor (TF3SF2) revealed a significant main effect of Response Rating, $\left[F_{(1,16)}=11.96, p=0.003, \eta^{2}=\right.$ $0.43]$. Overall, factor scores were more pronounced for responses that were subjectively perceived as correct. A statistical trend was found for the main effect Response Type, $\left[F_{(1,16)}=3.10\right.$, $\left.p=0.098, \eta^{2}=0.162\right]$, indicating also more pronounced factor scores for correct than for incorrect responses. The interaction was not significant, $F<1.2$. However, while factor scores were more pronounced for perceived than for unperceived correct responses, $p=0.014$, the difference between perceived and unperceived errors was not significant, $p=0.17$.

The first factor corresponding to the Pe (TF2SF1, Figure 3A), had a centro-parietal distribution and uniquely accounted for $2.27 \%$ of the variance in the solution whereas the second factor (TF2SF2, Figure 3B) showed a fronto-central distribution

A TF2SF1: centro-parietal factor
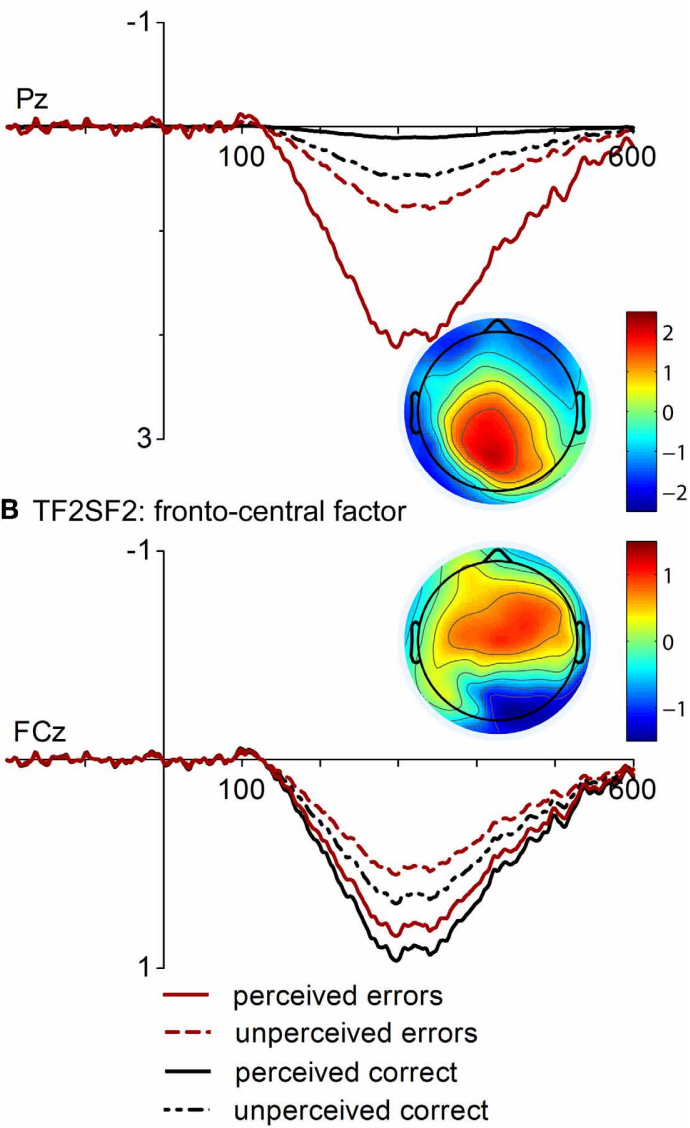

FIGURE 3 | "Virtual ERPs” and topographic maps (at $300 \mathrm{~ms}$ ) of the spatial factors contributing to $\mathrm{Pe}$. The waveforms are presented at electrodes $\mathrm{Pz}$ and $\mathrm{FC} z$ where factor scores were at maximum. Waveforms and topographies of the centro-parietal factor (A. TF2SF1) and of the fronto-central factor at $\mathrm{FC} z$ (B. TF2SF2) are depicted. 
and accounted for $1.49 \%$ of variance. The analysis of the centro-parietal factor (TF2SF1) showed significant main effects of Response Type, $\left[F_{(1,16)}=14.78, p=0.001, \eta^{2}=0.48\right]$, and Response Rating, $\left[F_{(1,16)}=13.24, p=0.002, \eta^{2}=0.45\right]$. These main effects were further specified by their significant interaction, $\left[F_{(1,16)}=6.13, p=0.025, \eta^{2}=0.28\right]$. Follow-up comparisons revealed that factor scores were more pronounced for both perceived and unperceived errors. However, the effect of Response Rating was significant for incorrect, but not for correct responses, $p=0.001$ vs. $p=0.183$. Factor scores of ERPs elicited by unperceived errors did not differ from those of unperceived correct responses, $p=0.432$. Finally, the analysis of the fronto-central factor of the Pe (TF2SF2) yielded no significant main effects but a significant interaction of Response Type and Response Rating, $\left[F_{(1,16)}=6.05, p=0.026, \eta^{2}=0.27\right]$. While an effect of Response Rating was found neither for incorrect nor for correct responses, a statistical significant difference between perceived correct responses and unperceived errors was found, $p=0.045$.

\section{DISCUSSION}

The present study investigated error awareness effects in a choice selection task in which errors are caused by perceptual difficulty instead of impulsive responding like in tasks used in most of the error awareness studies so far. The question was whether ERN and Pe amplitudes would be sensitive to the awareness of response correctness. Importantly, the main objective was to disentangle overlapping electrophysiological activity captured in the Pe by means of temporospatial PCA. Thereby, the sensitivity of the Pe subcomponents to error awareness was examined. To this end, a visual discrimination task with three difficulty levels and subsequent accuracy judgments was applied to elicit perceived and unperceived errors as well as perceived and unperceived correct responses. Although it was not possible to examine awareness effects for each difficulty level separately, we obtained a sufficient number of perceived and unperceived incorrect and correct responses when task difficulty was not considered.

ERP results based on classical amplitude analysis indicate that the ERN following errors was significantly larger than the negativity following correct responses. There was no difference in ERN size between perceived and unperceived errors, which is consistent with earlier studies (Nieuwenhuis et al., 2001; Endrass et al., 2005, 2007; O’Connell et al., 2007; Shalgi et al., 2009; Dhar et al., 2011; Hughes and Yeung, 2011), but inconsistent with more recent reports showing an amplitude reduction for unperceived errors (Maier et al., 2008; Hewig et al., 2011; Wessel et al., 2011). In fact, the current study revealed numerically identical ERN amplitudes for both error types. With regard to correct responses, CRNs tended to be larger for correct responses falsely judged as errors compared to perceived correct responses (Scheffers and Coles, 2000). Although the current data do not support a variation of ERNs with perceived incorrectness, the increase of the CRN with subjective error perception supports the view that the early response-related negativity might be important for the emergence of error awareness. While no distinction between error types was found the increase of the CRN with error perception indicates that the ERN might qualify as a necessary but not as a sufficient precondition for error awareness.
The PCA revealed two relevant temporospatial factors in the time range of response-related negativities: the first was characterized by a central negativity and the second by a frontal negativity and a parietal positivity. This factor solution was already shown in an earlier analysis of the current dataset which examined the effect of task difficulty and response correctness (Endrass et al., 2012). In that study, the central factor varied with response correctness and difficulty, while the frontal factor was not sensitive to task manipulations. In the current study the central factor also varied with response correctness and a trend for an interaction with perceived accuracy was found. This interaction indicated that factor scores varied with error perception on correct trials, but not on incorrect ones. Factor scores were larger for correct responses that were falsely considered as incorrect. These results indicate that the central factor is not only sensitive to the distinction between errors and correct responses but is also modulated by false error perception on correct responses. Thus, the modulation of the central factor is in accordance with ERP findings and might represent the underlying activity that modulates responserelated negativity amplitudes. Furthermore, the variation with response correctness and its sensitivity to error perception on correct trials further supports the idea that this factor represents specific aspects of error processing. This conclusion cannot be drawn for the frontal factor that varied only with perceived accuracy and was most pronounced for perceived correct responses. Since an influence of response correctness was also absent in our previous analysis, this factor was interpreted to represent an outcome-independent monitoring process contributing to both ERP components (Endrass et al., 2012). Certainly, more studies are needed to thoroughly understand the function of this component.

In contrast to response-related negativities, the error positivity, determined as conventional amplitude measure, showed a distinct variation with error awareness (Nieuwenhuis et al., 2001; Endrass et al., 2005, 2007; O'Connell et al., 2007; Shalgi et al., 2009; Dhar et al., 2011; Hughes and Yeung, 2011; Wessel et al., 2011). The current study supports these findings and shows that a variation of the Pe can also be found in a task where errors are caused by perceptual difficulty instead of failed response inhibition towards an imperative stimulus (Hughes and Yeung, 2011). Importantly, the Pe varied not only between perceived and unperceived errors but was also more positive for unperceived compared to perceived correct responses. Therefore, the Pe may represent a gradual measure for error awareness with its amplitude being most pronounced for perceived errors and least pronounced for perceived correct responses. This view is compatible with the conclusion drawn by Hewig and colleagues who found a similar modulation of the Pe amplitude by subjective correctness (Hewig et al., 2011). Regarding the idea that multiple changes in the salience network accumulate to the conscious perception of an error (Ullsperger et al., 2010), it seems that the modulation of the error positivity with subjective error perception on correct trials activates the salience network which leads to false error detection on correct trails. Although the current study found a variation of the Pe with false error perception on correct trials, the Pe in that condition was smaller compared to perceived errors. This indicates that subjective error perception 
may not depend on the presence of a pronounced Pe. Therefore, the current data are consistent with the assumption that the Pe, like the ERN, represents an internal error signal which is related to error processing and potentially to subjective error awareness, but it is not an all-or-nothing process that triggers error perception. Instead, error awareness might arise from multiple inputs at various stages during error processing (Steinhauser and Yeung, 2010; Ullsperger et al., 2010).

PCA analysis disentangled the error positivity into two temporospatial PCA factors as in the previous analysis of the present data (Endrass et al., 2012). Like in the previous analysis, a modulation of the centro-parietal factor with response correctness was found. Interestingly, the current study revealed an effect of subjective awareness on this same factor. The largest factor scores were found for perceived errors and smallest for perceived correct responses, while unperceived errors and unperceived correct responses did not differ and layed in-between. This modulation is fairly consistent with the results at ERP level suggesting that this factor represents the underlying process that triggers error awareness effects in the Pe. A centro-parietal factor was also identified in a previous PCA study. Therefore, the current study supports the assumption that the Pe represents a P300-like component to error commission (Arbel and Donchin, 2009). This component varied with error significance (Arbel and Donchin, 2009) and with error awareness in the current study. The modulation of the centro-parietal component subsequent to error commission may reflect the importance of an error and thus the saliency of that event. Consequently, the current PCA results support the idea that the Pe reflects the activity of a salience network that leads to error detection.

Post-error slowing, i.e., response time slowing following incorrect responses depended on subjective accuracy ratings and was more pronounced following perceived than unperceived errors. Therefore, it was argued that post-error slowing depended on the process reflected by the Pe and is also elicited by perceived errors (Nieuwenhuis et al., 2001; Endrass et al., 2007; Klein et al., 2007; Wessel et al., 2011). However, the current study not only revealed post-response slowing following perceived errors but also following unperceived correct responses. Therefore the slowing of response times in the subsequent trial occurred only when the previous response was perceived as incorrect independently of the objective correctness of that response (Hewig et al., 2011). Thus, it seems that post-error slowing occurred without a pronounced Pe in case of false error perception on correct trials. An alternative account considers post-error slowing as automatic behavioral adjustment by implementing compensatory cognitive control in the medio-frontal cortex (Botvinick et al., 2001; Gehring and Fencsik, 2001; Debener et al., 2005). However, the current data are also incompatible with that interpretation since unperceived errors elicited an ERN but were not followed by post-error slowing. A recent study found that post-response slowing occurred as a consequence to infrequent events irrespective of response correctness (Notebaert et al., 2009). Thus, it was considered as a slowing of task-relevant processing caused by an orienting response towards unexpected events. Although the current data cannot help to disentangle whether post-response slowing is an adaptive mechanism to prevent future errors or a distortion of stimulus processing leading to slower responses, it seems that this process is related to perceived incorrectness of a response rather than an automatic adjustment process outside of conscious error perception.

Whereas subsequent response times were influenced by a subjective error perception, post-error correctness was only influenced by effective error commission. Therefore, it might be assumed that the ERN which was present for perceived and unperceived errors is more closely related to post-error correctness. This relation appears independent of subjective error awareness. Thus, the current data may suggest that ERN and Pe reflect two partially independent error monitoring mechanisms leading to different adjustments at the behavioral level. One internal mechanism reflected in the ERN that detects errors and initiates subsequent adjustment of cognitive control improving correctness of a subsequent action. This adjustment might be accomplished outside of awareness but nevertheless related to automatic error processing, like increased theta coupling between the medial and lateral prefrontal cortex (Cavanagh et al., 2009), autonomic changes (Wessel et al., 2011), or the later error positivity. The second mechanism might be reflected in the Pe or, more specifically, its centro-parietal subcomponent. It is conceived as a P300-like component to motivationally salient events, i.e. to perceived incorrect responses. Subjective error perception though appears not the only modulator of the Pe since it was reduced in case of false error perception on correct trials. Thus, subjective error awareness may depend on gradual inputs during error processing like changes ERN, Pe, or autonomic response.

Possible limitations of the current study should be noted. First, error perception rates were quite low. Only $51.5 \%$ of the errors were recognized by the participants. However, similar rates were found in other experiments, like antisaccade tasks (Nieuwenhuis et al., 2001; Endrass et al., 2007). In addition, only $6.8 \%$ of the correct responses were falsely perceived as errors which leads to an acceptable discrimination accuracy $\left(d^{\prime}=1.52\right)$ suggesting that participants were able to judge response accuracy. Second, we were not able to separately analyze error awareness and task difficulty. Although both perceived and unperceived errors and correct responses were present at all difficulty levels, the amount of misclassifications, i.e., incorrect perceptions of the responses, varied with perceptual difficulty, especially for correct responses. Because more trials were presented in the easy condition the numbers of committed easy and difficult errors within perceived and unperceived errors did not differ. In addition, the analysis of a subsample of participants who had more than six perceived and unperceived errors in the easy and the difficult condition showed that an effect of error awareness was present for the Pe in both difficulty levels, but no variation of the ERN was found. Hence, the current findings of a difference between perceived and unperceived errors appear not to be caused by unequal amounts of easy and difficult trials. Nevertheless, the question whether the error awareness effect on Pe amplitudes is independent of task difficulty needs further examination.

To summarize, the current study replicates previous findings and demonstrates that subjective error awareness selectively modulates Pe but not ERN amplitudes. The temporospatial PCA identified two underlying factors for both ERP components. 
The factors in the time range of ERN were characterized by a central and a frontal topography. While the central factor putatively reflecting error processing was modulated by objective response correctness, it was unrelated to subjective error perception. The frontal factor which appears to reflect outcome-independent monitoring (Endrass et al., 2012) varied with subjective awareness irrespective of actual response correctness. The PCA factors underlying the Pe had a fronto-central and a centro-parietal distribution. Only the latter factor was related to error awareness: a selective enhancement was found for perceived errors compared to all other response types. Taken together these results support the functional distinctions of subcomponents of both the ERN and the Pe. The PCA results suggest that Pe modulations by experimental conditions are mostly due to variations of the centro-parietal subcomponent. Therefore, the Pe may represent a P300-like response reflecting motivational significance of an error and saliency processing.

\section{ACKNOWLEDGMENTS}

This work was supported by the German Research Foundation (EN 906/1-1).

\section{REFERENCES}

Arbel, Y., and Donchin, E. (2009). Parsing the componential structure of post-error ERPs: a principal component analysis of ERPs following errors. Psychophysiology 46, 1179-1189.

Berg, P., and Scherg, M. (1994). A multiple source approach to the correction of eye artifacts. Electroencephalogr. Clin. Neurophysiol. 90, 229-241.

Botvinick, M. M., Braver, T. S., Barch, D. M., Carter, C. S., and Cohen, J. D. (2001). Conflict monitoring and cognitive control. Psychol. Rev. 108, 624-652.

Brown, J. W., and Braver, T. S. (2007). Risk prediction and aversion by anterior cingulate cortex. Cogn. Affect. Behav. Neurosci. 7, 266-277.

Brown, J. W., and Braver, T. S. (2008). A computational model of risk, conflict, and individual difference effects in the anterior cingulate cortex. Brain Res. 1202, 99-108.

Cavanagh, J. F., Cohen, M. X., and Allen, J. J. (2009). Prelude to and resolution of an error: EEG phase synchrony reveals cognitive control dynamics during action monitoring. J. Neurosci. 29, 98-105.

Critchley, H. D., Tang, J., Glaser, D., Butterworth, B., and Dolan, R. J. (2005). Anterior cingulate activity during error and autonomic response. Neuroimage 27, 885-895.

Critchley, H. D., Wiens, S., Rotshtein, P., Ohman, A., and Dolan, R. J. (2004). Neural systems supporting interoceptive awareness. Nat. Neurosci. 7, 189-195.

Debener, S., Ullsperger, M., Siegel, M., Fiehler, K., Von Cramon, D. Y., and Engel, A. K. (2005). Trialby-trial coupling of concurrent electroencephalogram and functional magnetic resonance imaging identifies the dynamics of performance monitoring. J. Neurosci. 25, 11730-11737.
Dehaene, S., Posner, M. I., and Tucker, D. M. (1994). Localization of a neural system for error detection and compensation. Psychol. Sci. 5, 303-305.

Dhar, M., Wiersema, J. R., and Pourtois, G. (2011). Cascade of neural events leading from error commission to subsequent awareness revealed using EEG source imaging. PLOS ONE 6:e19578. doi: 10.1371/journal.pone.0019578

Dien, J. (2010a). The ERP PCA toolkit: an open source program for advanced statistical analysis of event-related potential data. J. Neurosci. Methods 187, 138-145.

Dien, J. (2010b). Evaluating two-step PCA of ERP data with Geomin, Infomax, Oblimin, Promax, and Varimax rotations. Psychophysiology 47, 170-183.

Dien, J., Beal, D. J., and Berg, P. (2005). Optimizing principal components analysis of event-related potentials: matrix type, factor loading weighting, extraction, and rotations. Clin. Neurophysiol. 116, 1808-1825.

Dien, J., and Frishkoff, G. (2005). "Principal components analysis of event-related potential datasets," in Event-Related Potentials: A Methods Handbook, ed T. C. Handy (Cambridge, MA: The MIT Press), 189-208.

Dien, J., Michelson, C. A., and Franklin, M. S. (2010). Separating the visual sentence N400 effect from the P400 sequential expectancy effect: cognitive and neuroanatomical implications. Brain Res. 1355, 126-140.

Endrass, T., Franke, C., and Kathmann, N. (2005). Error awareness in a saccade countermanding task. J. Psychophysiol. 19, 275-280.

Endrass, T., Klawohn, J., Gruetzmann, R., Ischebeck, M., and Kathmann, N. (2012). Response-related negativities following correct and incorrect responses: evidence from a temporospatial principal component analysis. Psychophysiology 49, 733-743.
Endrass, T., Reuter, B., and Kathmann, N. (2007). ERP correlates of conscious error recognition: aware and unaware errors in an antisaccade task. Eur. J. Neurosci. 26, 1714-1720.

Falkenstein, M., Hohnsbein, J., Hoormann, J., and Blanke, L. (1991). Effects of crossmodal divided attention on late ERP components: II. Error processing in choice reaction tasks. Electroencephalogr. Clin. Neurophysiol. 78, 447-455.

Falkenstein, M., Hoormann, J., Christ, S., and Hohnsbein, J. (2000). ERP components on reaction errors and their functional significance: a tutorial. Biol. Psychol. 51, 87-107.

Falkenstein, M., Hoormann, J., and Hohnsbein, J. (1999). ERP components in Go Nogo tasks and their relation to inhibition. Acta Psychol. 101, 267-291.

Ford, J. M. (1999). Schizophrenia: the broken P300 and beyond. Psychophysiology 36, 667-682.

Foti, D., Hajcak, G., and Dien, J. (2009). Differentiating neural responses to emotional pictures: evidence from temporal-spatial PCA. Psychophysiology 46, 521-530.

Foti, D., Weinberg, A., Dien, J., and Hajcak, G. (2011). Event-related potential activity in the basal ganglia differentiates rewards from nonrewards: temporospatial principal components analysis and source localization of the feedback negativity. Hum. Brain Mapp. 32, 2207-2216.

Gehring, W. J., and Fencsik, D. E. (2001). Functions of the medial frontal cortex in the processing of conflict and errors. J. Neurosci. 21, 9430-9437.

Gehring, W. J., Goss, B., Coles, M. G. H., Meyer, D. E., and Donchin, E. (1993). A neural system for error-detection and compensation. Psychol. Sci. 4, 385-390.

Hajcak, G., Mcdonald, N., and Simons, R. F. (2003). To err is autonomic error-related brain potentials, ANS activity, and post-error compensatory behavior. Psychophysiology 40, 895-903.

Herrmann, M. J., Rommler, J., Ehlis, A. C., Heidrich, A., and Fallgatter, A. J. (2004). Source localization (LORETA) of the error-relatednegativity (ERN/Ne) and positivity (Pe). Cogn. Brain Res. 20, 294-299.

Hester, R., Foxe, J. J., Molholm, S., Shpaner, M., and Garavan, H. (2005). Neural mechanisms involved in error processing: a comparison of errors made with and without awareness. Neuroimage 27, 602-608.

Hewig, J., Coles, M. G., Trippe, R. H., Hecht, H., and Miltner, W. H. (2011). Dissociation of Pe and ERN/Ne in the conscious recognition of an error. Psychophysiology $48,1390-1396$

Holroyd, C. B., and Coles, M. G. (2002). The neural basis of human error processing: reinforcement learning, dopamine, and the errorrelated negativity. Psychol. Rev. 109, 679-709.

Hughes, G., and Yeung, N. (2011). Dissociable correlates of response conflict and error awareness in error-related brain activity. Neuropsychologia 49, 405-415.

Kayser, J., and Tenke, C. E. (2005). Trusting in or breaking with convention: towards a renaissance of principal components analysis in electrophysiology. Clin. Neurophysiol. 116, 1747-1753.

Klein, T. A., Endrass, T., Kathmann, N., Neumann, J., Von Cramon, D. Y., and Ullsperger, M. (2007). Neural correlates of error awareness. Neuroimage 34, 1774-1781.

Leuthold, H., and Sommer, W. (1999). ERP correlates of error processing in spatial S-R compatibility tasks. Clin. Neurophysiol. 110, 342-357.

Maier, M., Steinhauser, M., and Hubner, R. (2008). Is the errorrelated negativity amplitude related 
to error detectability? Evidence from effects of different error types. J. Cogn. Neurosci. 20, 2263-2273.

Nieuwenhuis, S., Aston-Jones, G., and Cohen, J. D. (2005). Decision making, the P3, and the locus coeruleus-norepinephrine system. Psychol. Bull. 131, 510-532.

Nieuwenhuis, S., Ridderinkhof, K. R., Blow, J., Band, G. P. H., and Kok, A. (2001). Error-related brain potentials are differentially related to awareness of response errors: evidence from an antisaccade task. Psychophysiology 38, 752-760.

Notebaert, W., Houtman, F., Opstal, F. V., Gevers, W., Fias, W., and Verguts, T. (2009). Post-error slowing: an orienting account. Cognition 111, 275-279.

O'Connell, R. G., Dockree, P. M., Bellgrove, M. A., Kelly, S. P., Hester, R., Garavan, H., Robertson, I. H., and Foxe, J. J. (2007). The role of cingulate cortex in the detection of errors with and without awareness: a high-density electrical mapping study. Eur. J. Neurosci. 25, 2571-2579.

Overbeek, T. J. M., Nieuwenhuis, S., and Ridderinkhof, K. R. (2005). Dissociable components of error processing-on the functional significance of the Pe Vis-a-vis the ERN/Ne. J. Psychophysiol. 19, 319-329.

Picton, T. W. (1992). The P300 wave of the human event-related potential. J. Clin. Neurophysiol. 9, 456-479.

Ridderinkhof, K. R., Ramautar, J. R., and Wijnen, J. G. (2009). To P(E) or not to $\mathrm{P}(\mathrm{E})$ : a P3-like ERP component reflecting the processing of response errors. Psychophysiology 46, 531-538.

Ridderinkhof, K. R., Ullsperger, M., Crone, E. A., and Nieuwenhuis, S. (2004). The role of the medial frontal cortex in cognitive control. Science 306, 443-447.

Scheffers, M. K., and Coles, M. G. H. (2000). Performance monitoring in a confusing world: errorrelated brain activity, judgments of response accuracy, and types of errors. J. Exp. Psychol. Hum. Percept. Perform. 26, 141-151.

Shalgi, S., Barkan, I., and Deouell, L. Y. (2009). On the positive side of error processing: error-awareness positivity revisited. Eur. J. Neurosci. 29, 1522-1532.

Steinhauser, M., and Yeung, N. (2010). Decision processes in human performance monitoring. J. Neurosci. 30, 15643-15653.
Ullsperger, M., Harsay, H. A., Wessel, J. R., and Ridderinkhof, K. R. (2010). Conscious perception of errors and its relation to the anterior insula. Brain Struct. Funct. 214, 629-643.

Ullsperger, M., Volz, K. G., and Von Cramon, D. Y. (2004). A common neural system signaling the need for behavioral changes. Trends Cogn. Sci. 8, 445-446; author reply 446-447.

Van Veen, V., and Carter, C. S. (2002). The timing of action-monitoring processes in the anterior cingulate cortex. J. Cogn. Neurosci. 14 593-602.

Vidal, F., Hasbroucq, T., Grapperon, J., and Bonnet, M. (2000). Is the 'error negativity' specific to errors? Biol. Psychol. 51, 109-128.

Vocat, R., Pourtois, G., and Vuilleumier, P. (2008). Unavoidable errors: a spatio-temporal analysis of time-course and neural sources of evoked potentials associated with error processing in a speeded task. Neuropsychologia 46, 2545-2555.

Wessel, J. R., Danielmeier, C., and Ullsperger, M. (2011). Error awareness revisited: accumulation of multimodal evidence from central and autonomic nervous systems. J. Cogn. Neurosci. 23, 3021-3036.
Yeung, N., Botvinick, M. M., and Cohen, J. D. (2004). The neural basis of error detection: conflict monitoring and the error-related negativity. Psychol. Rev. 111, 931-959.

Conflict of Interest Statement: The authors declare that the research was conducted in the absence of any commercial or financial relationships that could be construed as a potential conflict of interest.

Received: 17 January 2012; accepted: 31 May 2012; published online: 22 June 2012.

Citation: Endrass T, Klawohn J, Preuss J and Kathmann N (2012) Temporospatial dissociation of $\mathrm{Pe}$ subcomponents for perceived and unperceived errors. Front. Hum. Neurosci. 6:178. doi: 10.3389/fnhum.2012.00178 Copyright (c) 2012 Endrass, Klawohn, Preuss and Kathmann. This is an openaccess article distributed under the terms of the Creative Commons Attribution Non Commercial License, which permits non-commercial use, distribution, and reproduction in other forums, provided the original authors and source are credited. 